\title{
Fibrillated cellulose in heterophase polymerization of nanoscale poly(methyl methacrylate) spheres
}

\author{
Franziska Grüneberger $^{1,2}$ • Anja Huch ${ }^{1}$. Thomas Geiger ${ }^{1}$ - Tanja Zimmermann ${ }^{1}$. \\ Philippe Tingaut ${ }^{1}$
}

Received: 29 March 2016 /Revised: 31 May 2016 / Accepted: 31 May 2016 /Published online: 11 June 2016

(C) Springer-Verlag Berlin Heidelberg 2016

\begin{abstract}
Nanofibrillated cellulose (NFC) was used as biobased stabilizing agent in the heterophase polymerization of methyl methacrylate (MMA) to nanoscale poly(methyl methacrylate) (PMMA) spheres. NFC/MMA suspensions at different NFC/MMA ratios were prepared in water, and the suspension stability was evaluated before the subsequent polymerization. The resulting polymerization products as well as products from several control experiments were analyzed via optical microscopy, SEM, and isolation experiments associated with thermogravimetric analysis. PMMA spheres had diameters in the range of $150-250 \mathrm{~nm}$ and were regularly distributed within the NFC network, whereas NFC acted as a stabilizer during the polymerization. The appearance of the resulting PMMA spheres within the NFC network was influenced by different factors, such as the reaction conditions, the initiator, and the solubility of the monomer.
\end{abstract}

Keywords Nanofibrillated cellulose (NFC) .

PMMA spheres · Heterophase polymerization $\cdot$ Nanoparticles

Electronic supplementary material The online version of this article (doi:10.1007/s00396-016-3899-2) contains supplementary material, which is available to authorized users.

Franziska Grüneberger

Franziska.Grueneberger@empa.ch

Thomas Geiger

Thomas.Geiger@empa.ch

1 Empa, Swiss Federal Laboratories for Materials Science and Technology, Applied Wood Materials, Ueberlandstrasse 129, 8600 Dübendorf, Switzerland

2 Institute for Building Materials (IfB), Wood Materials Science, ETH Zürich, Stefano-Franscini-Platz 3, 8093 Zürich, Switzerland

\section{Introduction}

There is a broad variety in applying particle-stabilized emulsions, e.g., in drug delivery systems, in cosmetic or food applications, and even for the preparation of porous materials [1]. Such systems, which are solely stabilized by solid (nano)particles, were initially described in the 1900s by Ramsden [2] and Pickering [3]. Suitable particles must be partially wettable by both the dispersed and the dispersing phases, which can also be reached by using functionalized particles. The advantage of particle stabilized over classical, surfactant-stabilized systems is the fact that the particles are adsorbed irreversibly to the interface. They form rigid interface structures, since the adsorption energy of the particle to the interface is much higher than its thermal energy [4]. In contrast, surfactants may desorb quite easily from the interface, which might be disadvantageous concerning system stability.

The field of applications for particle-stabilized emulsions may be expanded by using solid particles to stabilize heterophase polymerizations, including emulsion, miniemulsion, dispersion, and suspension polymerization [5]. Different types of inorganic and organic particles were already used in heterophase polymerizations, e.g., silica, zinc oxide, clay, or magnetic particles as well as polymer latex particles [6-10]. Moreover, the stability of the polymerization system and the appearance of the resulting polymerization products are strongly influenced by the choice of suitable co-monomers, co-solvents, initiators, and other reaction parameters such as reaction conditions and $\mathrm{pH}$ or salt content $[5,11,12]$.

Nanocellulosic materials, namely nanofibrillated cellulose (NFC), microcrystalline or nanocrystalline cellulose (MCC/ NCC), and bacterial cellulose (BC), can also serve as novel bio-based particulate stabilizers. They were already used for the stabilization of emulsions and for the preparation of latex 
particles by miniemulsion polymerization for different applications as recently summarized in several reviews [13-15]. Nanocelluloses derived from cellulose, the most abundant biopolymer on earth, exhibit both hydrophilic and hydrophobic planes in their crystalline basic structure, and they can also be chemically modified due to the hydroxyl groups of their Dglycopyranose units [16].

Heterophase polymerization in presence of nanocelluloses was mainly reported for NCC. Ben Mabrouk polymerized latex particles in presence of NCC, whose emulsionstabilizing effect before the polymerization was improved by using either mediating surfactants or by surface silylation [17-19]. Other authors took profit of the polymerization approach to visualize their originally prepared oil-in-water $(\mathrm{O} /$ W) emulsions, which were stabilized by NCC. Therefore, the oil phase of the emulsions was replaced by styrene, which was subsequently polymerized to polystyrene particles in micrometer range, and thus, the appearance of the emulsions could be visualized by SEM [20]. In this way, different effects on the emulsion appearance and stability, such as the NCC aspect ratio, surface charge or modification, or the ionic strength, were assessed [21-25].

A few reports also exist on using NFC in particle-stabilized systems. For instance, O/W emulsions with soybean oil for food applications were stabilized with unmodified NFC and silylated, hydrophobized NFC-stabilized water-in-oil (W/O) emulsions [26, 27]. Further, heterophase polymerization in presence of NFC was reported by Littunen et al. [28], who surface-coated NFC by using a cerium initiator, and by Nikfariam et al. [29], who produced expandable NFCstabilized polystyrene (PS) beads.

In the present work, we prepared nanoscale poly(methyl methacrylate) (PMMA) spheres by emulsion polymerization solely stabilized by nanoscale NFC network. NFC was chosen as the solid stabilizer and methyl methacrylate (MMA) as the monomer, since only limited information is available on their combined use in heterophase polymerization systems. Before polymerization, the stability of aqueous suspensions of MMA and NFC at different NFC/MMA ratios was evaluated. The subsequently obtained PMMA spheres were homogeneously distributed within the NFC network, and the role of NFC, as well as the effect of the monomer solubility, the reaction conditions, and different initiators on the resulting NFC/PMMA products, was evaluated.

\section{Materials and methods}

\section{Materials}

Nanofibrillated cellulose (NFC) was obtained by mechanical disintegration of a never dried, totally chlorine-free softwood kraft pulp (TCF, Zellstoff Stendal, Germany) as already reported before [30] and was used in the form of a gel-like aqueous suspension $(2.5 \mathrm{wt} \%)$. The NFC was not chemically modified for these experiments, and zeta potential measurements (not shown) revealed a negative surface charge due to the hydroxyl groups of the cellulose. The monomer methyl methacrylate (MMA; Merck KGaA) was purified prior to polymerization via extraction with $5 \mathrm{wt} \%$ sodium hydroxide solution and subsequent extraction with water and saturated sodium chloride solution. The initiators 2,2'-azobis(2methylpropionitrile) (AIBN) and potassium persulfate (KPS; both Sigma-Aldrich) and the surfactants sodium dodecyl sulfate (SDS) and cetyltrimethylammonium bromide (CTAB, both Merck KGaA) were used as received.

\section{Methods}

\section{Suspension stability}

Before polymerization, the stability of suspensions, containing NFC and MMA, was assessed. Therefore, NFC at different concentrations $(0.05,0.1,0.25,0.5 \mathrm{wt} \%$ in final mixture) was homogeneously dispersed in the desired amount of water by using a homogenizer (Ultra-Turrax T25; S25N-25F dispersing unit, IKA-Werke). The monomer MMA ( $5 \mathrm{wt} \%$ in final mixture) was added to the aqueous NFC suspensions, and the resulting mixtures were homogenized for $2 \mathrm{~min}$ at $14,000 \mathrm{rpm}$ (homogenizer) at RT. The thus prepared suspensions will be referred to as NFC/MMA suspensions. Analogous suspensions of neat NFC in water were prepared for comparison. After preparation, the suspensions were stored in test tubes and visually assessed after $1 \mathrm{~h}$ and up to 11 weeks.

\section{Polymerization of MMA to NFC/PMMA products}

Table 1 contains the typical formulations of the suspensions for the polymerization of MMA in presence of NFC. The aqueous phase contained NFC at different concentrations, and the monomer or organic phase contained the monomer MMA at a constant concentration of $5 \mathrm{wt} \%$ and the initiator AIBN. The reaction mixtures were prepared as described for the suspension stability tests, while the final homogenization step was performed under a constant nitrogen flow. The reaction flasks were sealed with a septum and were further purged with nitrogen for at least 10 min prior to the polymerization. The reaction flasks were placed in an oven, and the polymerization $\left(80^{\circ} \mathrm{C}, 4 \mathrm{~h}\right)$ was conducted without stirring, which is further referred to as static conditions. It should be considered that the reaction flasks had to be deflated regularly during polymerization to avoid overpressure. NFC/PMMA suspensions were further used as polymerized.

The polymerization was additionally conducted under dynamic conditions. Therefore, the reaction flask was equipped 
Table 1 Compositions of NFC/ MMA suspensions for polymerization

\begin{tabular}{llcccc}
\hline NFC/PMMA ratios & & $1: 100$ & $1: 50$ & $1: 20$ & $1: 10$ \\
\hline Compounds & & Content (wt\%) & & \\
Aqueous phase & Water & 94.7 & 94.65 & 94.5 & 94.25 \\
& NFC $^{\mathrm{a}}$ & 0.05 & 0.1 & 0.25 & 0.5 \\
Organic phase & MMA or styrene & & & & \\
Initiator & AIBN (organic phase) or KPS (aqueous phase) & $0.05^{\mathrm{c}}$ & & & \\
\hline
\end{tabular}

${ }^{\text {a }}$ Excluded for polymerization of unstabilized PMMA in control experiment

${ }^{\mathrm{b}}$ Styrene for control experiment

${ }^{\mathrm{c}} 1 \mathrm{wt} \%$ relative to monomer with a condenser and polymerization ran under stirring with a magnetic stirrer $\left(80^{\circ} \mathrm{C}, 4 \mathrm{~h}\right)$. Polymerization was moreover performed while using the water.

\section{Control experiments for polymerization}

Control experiments were accomplished to show the influence of NFC and of the monomer MMA during polymerization. Unstabilized PMMA was polymerized by AIBN initiation in absence of NFC or any other stabilizing agent under static conditions. Physically blended NFC/PMMA suspensions were prepared by mixing the unstabilized PMMA together with NFC by using the homogenizer ( 2 min, 14,000 rpm) at the same NFC/PMMA ratios as given in Table 1. Furthermore, MMA was replaced by styrene and polymerized by AIBN initiation in presence of NFC under static conditions.

\section{Isolation experiments}

Non-bounded, free PMMA spheres were isolated by centrifugation from the NFC/PMMA suspensions, which were obtained according to the standard recipe (AIBN, static) and according to one control experiment (KPS, dynamic). Therefore, $10 \mathrm{~g}$ of the NFC/PMMA suspensions was centrifuged (5 min at 5000g; Rotina 380, Hettich) and the supernatant was discarded. The utilization of surfactants, SDS and $\mathrm{CTAB}$, respectively, was necessary during the subsequent re-dispersion/centrifugation steps to avoid the sedimentation of free PMMA spheres during centrifugation. Therefore, $30 \mathrm{ml}$ of a $17 \mathrm{mM}$ SDS or $30 \mathrm{ml}$ of a 2.2-mM CTAB solution in water was added to the precipitate and NFC/PMMA was redispersed by using the homogenizer at 10,000 rpm. The resulting mixtures were centrifuged again, and the supernatant was discarded. This re-dispersion/centrifugation step was repeated trice. Finally, the NFC/PMMA was washed with deionized water in order to remove all remaining surfactants and free PMMA spheres until the supernatant was clear after centrifugation. The resulting precipitate was diluted again with water to give the initial mass of $10 \mathrm{~g}$ and analyzed in suspension as well as freeze-dried powder via thermogravimetric analysis (TGA) analysis.

\section{Characterization}

Optical microscopy (Zeiss Stemi SV11) was used to evaluate the consistency of NFC/PMMA as suspension after polymerization or after the isolation experiments. Therefore, the suspensions were diluted to $0.01 \mathrm{wt} \% \mathrm{NFC}$.

SEM images of NFC/PMMA were recorded with an accelerating voltage of $5 \mathrm{kV}$ and a working distance of $5 \mathrm{~mm}$ (FEI Nova NanoSEM 230 instrument). Therefore, one drop of the diluted NFC/PMMA suspension ( $0.1 \mathrm{wt} \%$ NFC) was placed onto a mica plate laminated to a specimen holder. The drop was dried in vacuum and sputtered directly with a platinum layer of $8 \mathrm{~nm}$ (BAL-TEC AG, Lichtenstein).

Dynamic light scattering (DLS) was conducted to determine the PMMA particle size of neat PMMA reference spheres $(0.1 \mathrm{wt} \%)$ in water (Malvern Zetasizer Nano ZS).

TGA of non-isolated and isolated NFC/PMMA samples was conducted at a heating rate of $20^{\circ} \mathrm{C} / \mathrm{min}$ under nitrogen atmosphere up to $700{ }^{\circ} \mathrm{C}$ (PerkinElmer TGA7).

\section{Results and discussion}

\section{Suspension stability before polymerization}

In this work, a nanoscale NFC network was chosen to act as solid stabilizer in the heterophase polymerization of MMA. Before the polymerization, the appearance and the stability of suspensions consisting of MMA (dispersed phase, $5 \mathrm{wt} \%$ ) and NFC (stabilizer, 0-0.5 wt \%) in water (continuous phase) were evaluated (Fig. 1).

Stable and homogeneous NFC/MMA suspensions were formed directly after mechanical agitation for all investigated NFC concentrations (Fig. 1a) and reference samples with neat NFC (Fig. 1b). These suspensions did not change significantly during the first hour, while creaming, which is also called 
Fig. 1 Stability of NFC/MMA (a, c) and respective neat NFC suspensions $(\mathbf{b}, \mathbf{d})$ depending on the NFC concentration and on the storage time after suspension preparation

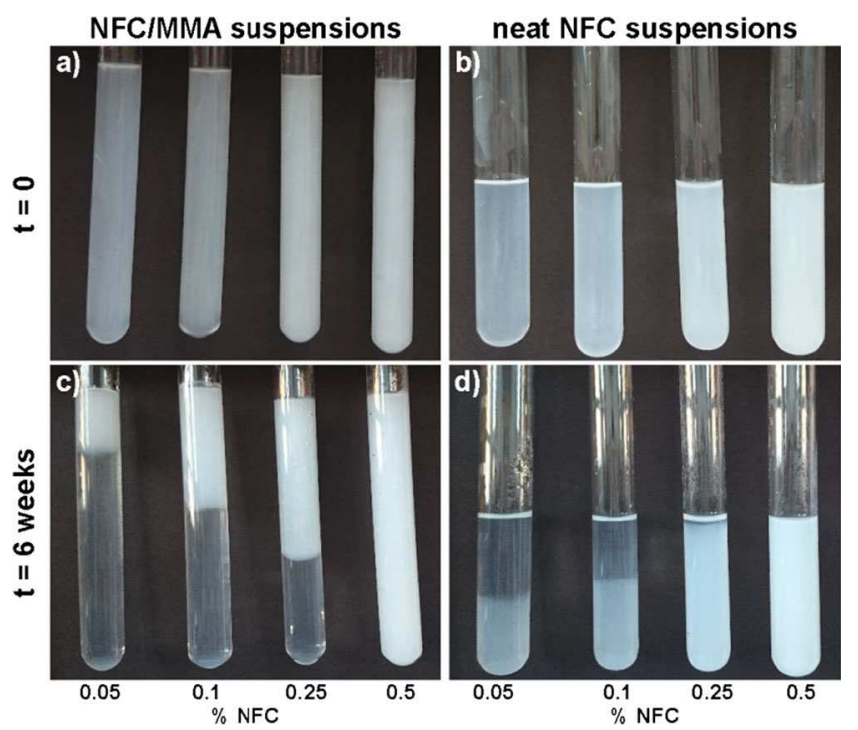

On the other hand, the suspension stability is further related to the comparably high solubility of MMA in water $(1.5 \mathrm{~g} / 100 \mathrm{ml})$, despite of its more hydrophobic character. Thus, MMA is not only dispersed but also, to a certain extent, dissolved in the aqueous phase. For this reason, distinct MMA droplets were not observed within the suspension via microscopy and the morphology of the suspensions was not visible, not even after staining the MMA with an oil-soluble dye. Furthermore, Liu et al. [36] suggested that the MMA/water interface is more indistinct due to the solubility of MMA, which enhances the stability of NFC/MMA suspensions. In their study, only oil phases, which were slightly soluble in water (MMA, ethyl acetate), were successfully stabilized with unmodified NCC in contrast to less soluble oil phases (i.e., toluene, hexane, styrene).

Interestingly, the used NFC was not able to stabilize higher amounts of MMA, since MMA phase-separated from the NFC/MMA suspensions already at $10 \mathrm{wt} \%$ MMA. Improved stability of higher amounts of MMA might be achieved either by an increased NFC concentration, by using chemically modified NFC, or by salt addition, which are reported approaches for improving the stabilization potential of NFC and, thus, the suspension stability [21, 37, 38].

\section{Polymerization of MMA in presence of NFC}

MMA emulsified at different NFC concentrations was then polymerized under varying reaction conditions (static/dynamic) with different initiators (AIBN/KPS). SEM images (Fig. 2a-d) of NFC/PMMA products polymerized under static conditions (no stirring) with AIBN revealed the presence of a NFC network, which was uniformly covered with spherical PMMA particles in the nanometer range. The majority of the 
Fig. 2 SEM images of NFC/ PMMA suspensions at increasing NFC concentrations during polymerization: a $0.05 \mathrm{wt} \%$ (inset: neat NFC), b $0.1 \mathrm{wt} \%$, c $0.25 \mathrm{wt} \%$, and $\mathbf{d} 0.5 \mathrm{wt} \%$ (inset: magnification) NFC; the MMA concentration was kept constant at $5 \mathrm{wt} \%$ (initiator: AIBN)
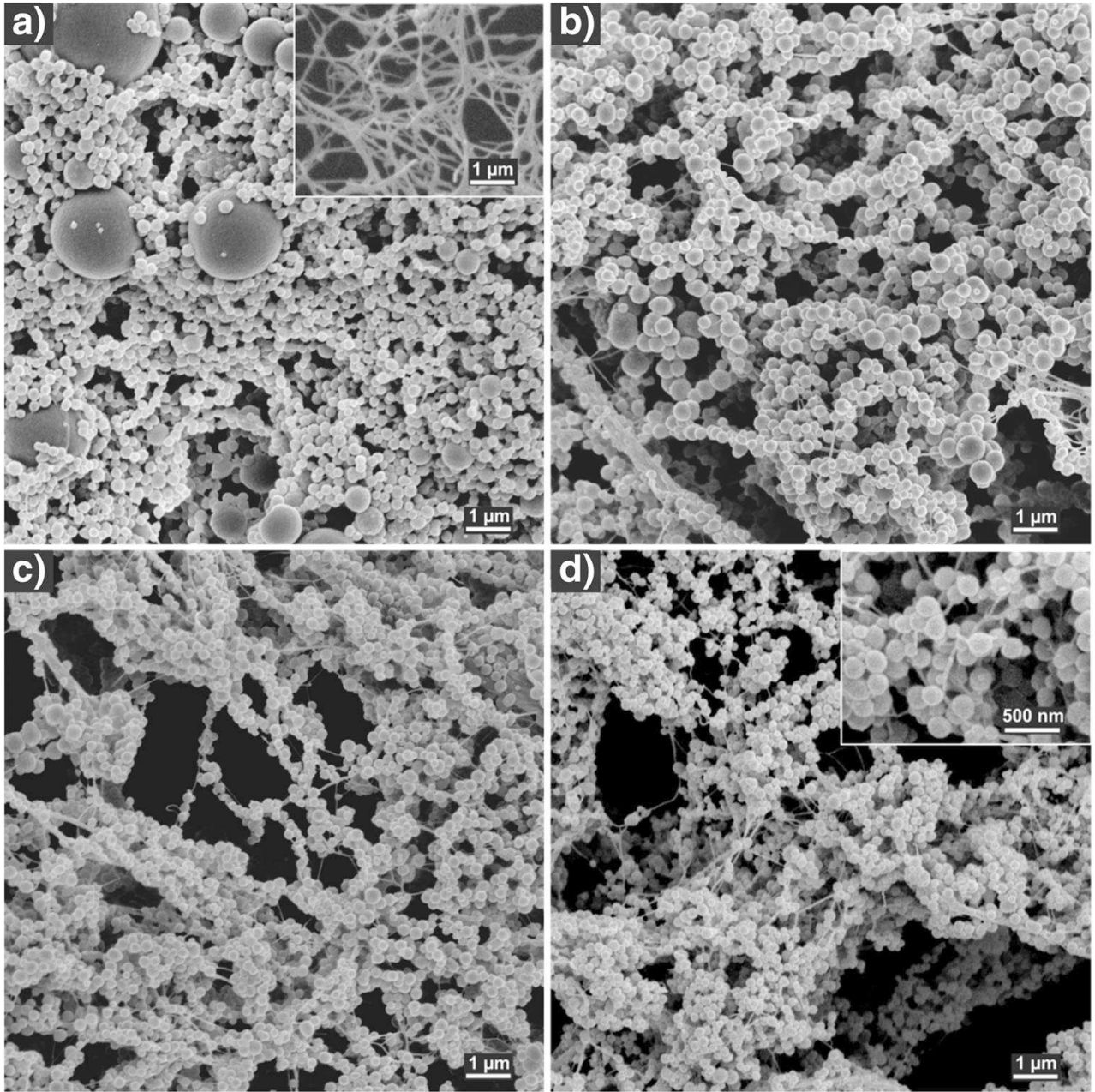

PMMA spheres displayed diameters from 150 to $250 \mathrm{~nm}$ at all investigated NFC concentrations. Few spheres with diameters in the micrometer range (up to $5 \mu \mathrm{m}$ ) were observed at the lowest NFC concentration ( $0.05 \mathrm{wt} \%$, Fig. $2 \mathrm{a})$, indicating that concentrations above $0.05 \mathrm{wt} \% \mathrm{NFC}$ are necessary to obtain a sufficient MMA dispersion. For comparison, a SEM image of the neat NFC network without PMMA is shown in the inset of Fig. 2a.

The formed PMMA spheres adhered to the NFC fibrils, most probably caused by non-covalent interactions between the NFC and PMMA, such as hydrogen bonds between the hydroxyl groups of the NFC and the carboxylic groups of the MMA. Additionally, NFC also exhibits hydrophobic planes in its structure, and therefore, an interaction with the MMA via hydrophobic van der Waals forces is assumed [16].

\section{Influence of reaction conditions and initiator}

Changing the reaction conditions from static (no stirring) to dynamic (stirring) and using the water-soluble initiator KPS instead of AIBN resulted in different NFC/PMMA products compared to the regular PMMA spheres adhering to NFC, which were described before.

Under dynamic conditions and at low NFC concentration $(0.05 \mathrm{wt} \%)$, large PMMA beads in millimeter range were formed (Fig. 3a), which were not present under static conditions (compare with Fig. s1a, supplementary information [SI]). At high NFC concentration (0.5 wt $\%$, Fig. $3 b)$, the NFC network was loosely covered with small, irregularly shaped PMMA particles and aggregates in micrometer range. Obviously, the kinetic energy, which was applied to the suspension via stirring, disturbs the weak, non-covalent interactions between NFC and (P)MMA. As a result, the formation of regularly shaped PMMA spheres on the NFC was inhibited and led to MMA coagulation. Thus, large PMMA beads were formed, since AIBN as an oil-soluble initiator nearly exclusively decomposes within the oil or monomer phase [39]. Consequently, a polymerization reaction only occurs in the monomer droplets, which is typical for the suspension polymerization, resulting in large polymer beads, or the miniemulsion polymerization, during which small, stabilized 
Fig. 3 Images from optical and SEM microscopy of NFC/PMMA suspensions polymerized under dynamic conditions at a low $(0.05 \mathrm{wt} \%)$ and b high $(0.5 \mathrm{wt} \%)$ NFC concentrations during polymerization (initiator: AIBN)
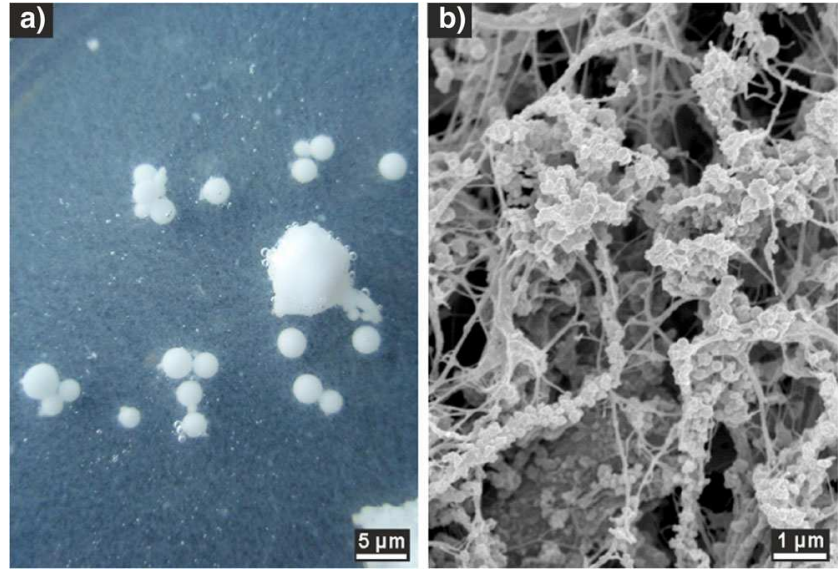

polymer particles are obtained. Since small, homogeneously distributed PMMA spheres instead of large PMMA beads were observed in the NFC network under static conditions, we think that a templating effect of the NFC and the solubility of the monomer are essential factors for the appearance of the NFC/PMMA products, which will be further discussed in the next sections.

Similar assumptions were done in the heterophase polymerization of MMA in presence of silica particles under static conditions [6]. There, static conditions resulted in raspberrylike PMMA particles decorated and stabilized, respectively, by silica particles due to weak PMMA-silica interactions. Dynamic conditions disrupted these weak interactions and finally separated PMMA particles, and aggregates were formed.

NFC/PMMA polymerization with the initiator KPS at low NFC content resulted in regular PMMA spheres $(176 \pm 18 \mathrm{~nm}$, values based on 50 measurements on Fig. $4 \mathrm{a})$, which were arranged like a mat of spheres on top of the NFC network (Fig. 4a). At higher NFC concentrations (Fig. 4b), NFC fibrils were mostly decorated with PMMA spheres, similar to the structures obtained with AIBN, and arrays of free PMMA spheres were still present. The reaction conditions (static/dynamic) did not have an influence on the obtained NFC/PMMA polymerized with KPS. We assume that by using the water-soluble initiator KPS, partially free PMMA spheres were formed, which did not adhere to the NFC. The water-soluble initiator KPS decomposes in the water phase and therefore promotes homogeneous nucleation and, thus, surfactant-free emulsion polymerization. There, only small polymer particles in nanometer range are formed, since the monomer MMA diffuses to the reaction sites in the aqueous phase and large MMA droplets rather serve as monomer reservoir than as nucleation site [40].

Our assumption of partially free PMMA spheres was further supported by the fact that the obtained NFC/PMMA suspensions were turbid and milky-white like a normal latex emulsion (compare Fig. s2a, SI) and by the fact that monodisperse PMMA particles were obtained in a control experiment without NFC (Fig. s3 B/D, SI), which is well known for surfactant-free emulsion polymerization [41]. Moreover, qualitative isolation experiments were conducted to support our assumption.

After isolation of free PMMA spheres from NFC/PMMA suspensions with a series of centrifugation and re-dispersion steps in presence of aiding surfactants, the NFC/PMMA polymerizates obtained with KPS were not turbid anymore (Fig. s2, SI) and larger NFC/PMMA flocks could be distinguished. The beforehand present array of free PMMA spheres was not visible anymore on the SEM images, only the PMMA spheres, which were regularly distributed in the NFC network, remained (Fig. s4c, SI). In contrast, the NFC/PMMA suspensions prepared with AIBN did not change their macroscopic and microscopic appearance significantly, which could be expected since the PMMA seemed to adhere to the NFC. Isolated and non-isolated samples were further subjected to thermogravimetric analysis, and the resulting derivative thermogravimetric (DTG) curves are presented in Fig. 5. A detailed peak assignment of the DTG curves is given in the SI (Fig. s4).

The DTG curves of the NFC/PMMA samples polymerized with KPS changed most significantly (Fig. 5a). PMMA degradation peaks at about 180 and $300{ }^{\circ} \mathrm{C}$ minimized or disappeared after the isolation experiments, which indicates a loss of PMMA in the sample, most probably due to removal of non-bound PMMA. When CTAB was used as aiding surfactant during the re-dispersion, a new peak appeared after the isolation experiment at $331-333{ }^{\circ} \mathrm{C}$. This peak is assigned to a $\mathrm{NFC/CTAB}$ assembly due to adsorbed cationic CTAB on the anionic NFC surface, since the same peak was observed for the NFC + CTAB control (Fig. s5, SI). After removal of PMMA, NFC became available for the adsorption of CTAB, and therefore, the new peak emerged. DTG curves of samples from polymerization with AIBN (Fig. 5b) differed less before 
Fig. 4 SEM images of NFC/

PMMA suspensions polymerized

with the initiator KPS at a low

$(0.05 \mathrm{wt} \%)$ and $\mathbf{b}$ high $(0.5 \mathrm{wt} \%)$

NFC content during

polymerization
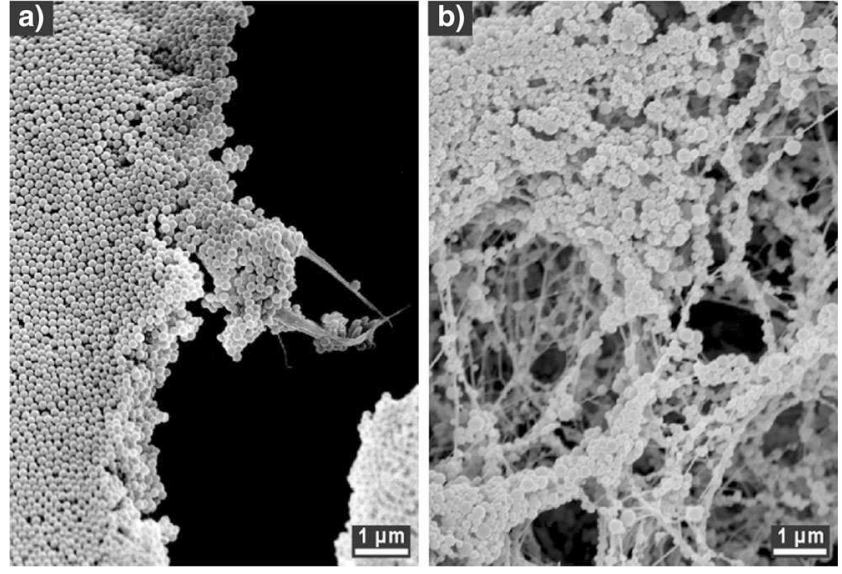

and after isolation; all PMMA degradation peaks remained and no additional peak appeared. This indicates that most PMMA spheres adhered to the NFC and remained in the NFC/PMMA suspension during isolation.

Next to the solubility of the initiators and, therefore, the dissimilar modes of heterogeneous polymerizations,
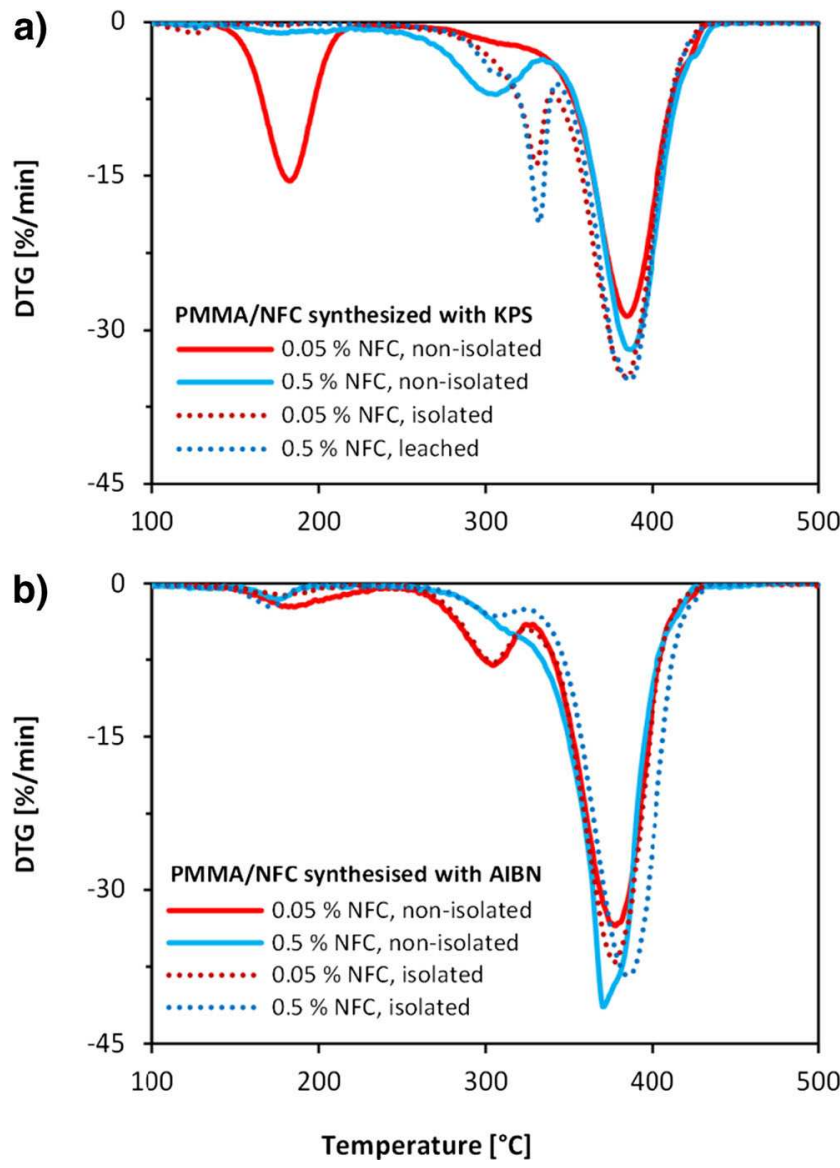

Fig. 5 DTG curves of NFC/PMMA products polymerized with KPS (a) and $\operatorname{AIBN}(\mathbf{b})$ before and after the isolation experiment with CTAB with non-isolated and isolated samples at low $(0.05 \mathrm{wt} \%)$ and high $(0.5 \mathrm{wt} \%)$ NFC concentrations during polymerization reaction the formation of free (KPS) and non-free (AIBN) PMMA spheres might be promoted by the unlike charges of the initiators. KPS is an anionic species and therefore carries the same negative charge as the NFC surface as determined by zeta potential measurements (not shown). Thus, repulsive forces between NFC and KPS exist, which promote the formation of free PMMA spheres rather than adhered spheres. In contrast, AIBN as non-ionic species does either not interact with the NFC surface at all, or, more probably, it builds up weak hydrophobic interactions with the hydrophobic planes of the NFC. Thus, the adhesion of formed PMMA spheres on the NFC fibrils is promoted. Analogue conclusions were drawn in literature for the polymerization of zinc oxide $(\mathrm{ZnO}) / \mathrm{PS}$ or silica/PMMA composites [7, 42].

In summary, the PMMA particle formation in the two different systems (AIBN or KPS initiated) can be described as follows (compare Fig. s6, SI): With the non-ionic initiator AIBN, PMMA spheres are formed by suspension polymerization. The initiator is dissolved in the monomer droplets, which are stabilized by the NFC fibrils. Polymer growth starts in the monomer droplets, and under static conditions, the resulting regularly formed polymer spheres adhere to the NFC fibrils due to assumed non-covalent, hydrophobic interactions. These interactions are possible between NFC and AIBN and the monomer units, respectively. With the anionic initiator KPS, PMMA spheres are formed by emulsion polymerization. KPS is dissolved in the aqueous phase, and monomer droplets, which serve as monomer reservoir, are stabilized by the NFC network within the suspension. The polymerization is initiated in the aqueous phase, and the growing polymer chains assemble to polymer particles. The resulting regularly formed polymer spheres are present either as free spheres due to the anionic initiator, which stabilizes the polymer spheres and which is repelled by the 

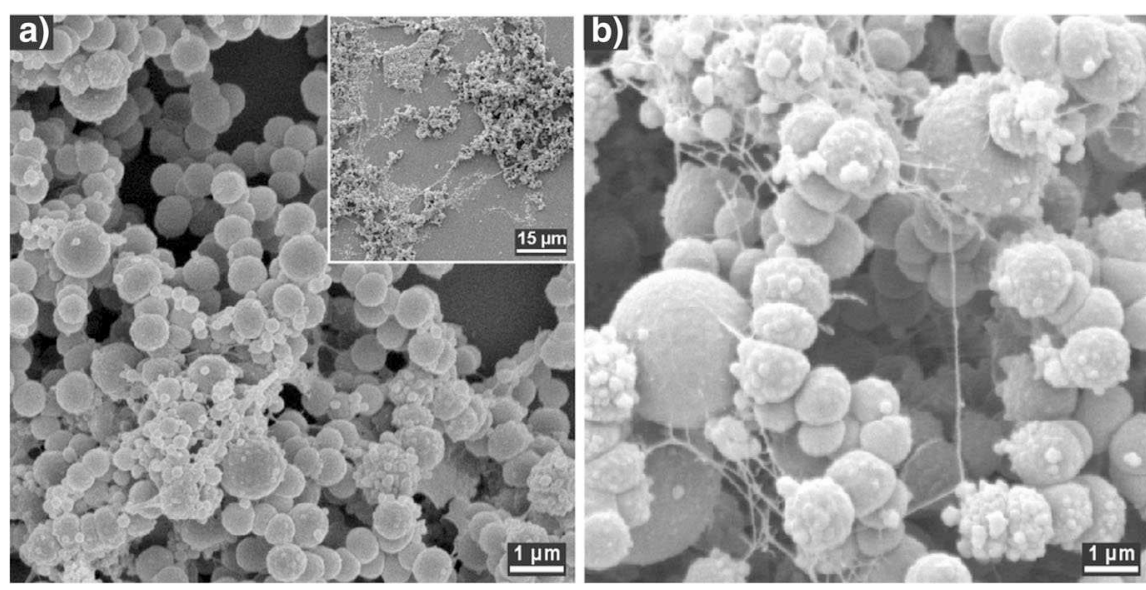

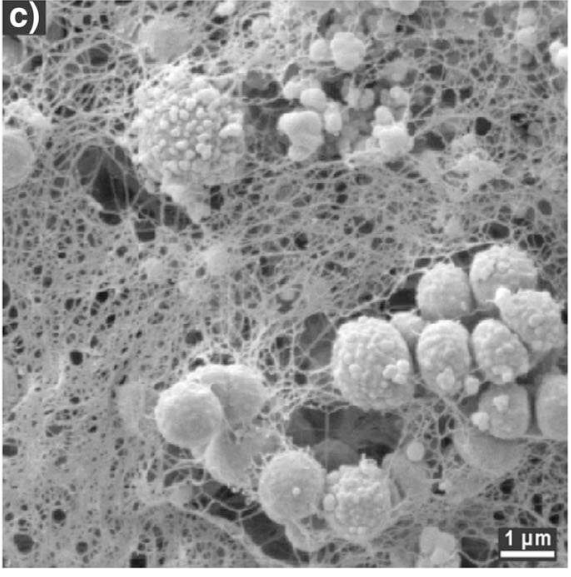

Fig. 6 SEM images of unstabilized PMMA particles and aggregates, respectively (a), and NFC/PMMA suspensions obtained by physical blending of unstabilized PMMA and NFC at two different NFC concentrations: $0.1 \mathrm{wt} \%$ (b) and $0.5 \mathrm{wt} \%$ (c); these images can be compared with the SEM images in Fig. 2b, d anionic NFC surface, or as spheres, which adhere to the NFC due to hydrophobic interactions between NFC and the monomer units.

\section{Stabilizing effect of NFC}

Since the obtained PMMA spheres appeared homogeneously distributed within the NFC network, NFC acted as the stabilizer or the carrier during the polymerization of MMA to PMMA. This assumption is based on the weak non-covalent interactions between NFC and MMA or AIBN, respectively, and the presence three-dimensional NFC structures as mechanical barriers against coagulation, which were both suggested before.

The stabilizing effect of NFC was supported by further control experiments. Therefore, unstabilized PMMA was polymerized under the same, static reaction conditions but in absence of NFC and subsequently physically blended with NFC. The NFC-free suspension consisted of large PMMA spheres with diameters mainly between 500 and $1000 \mathrm{~nm}$, which also formed macroscopic aggregates due to insufficient colloidal stability (Figs. 6a and s3C, SI). It has to be mentioned that an additional solid layer of PMMA was formed on top of the reaction flask during polymerization due to MMA phase separation and consequent bulk polymerization in presence of oil-soluble AIBN. This solid layer was removed before the physical blending.

The SEM images of the physically blended NFC/PMMA suspensions at two different NFC/PMMA ratios (Fig. 6b, c) revealed that PMMA spheres and their aggregates with a broad particle size distribution were irregularly distributed within or on top of the NFC network. This is in significant
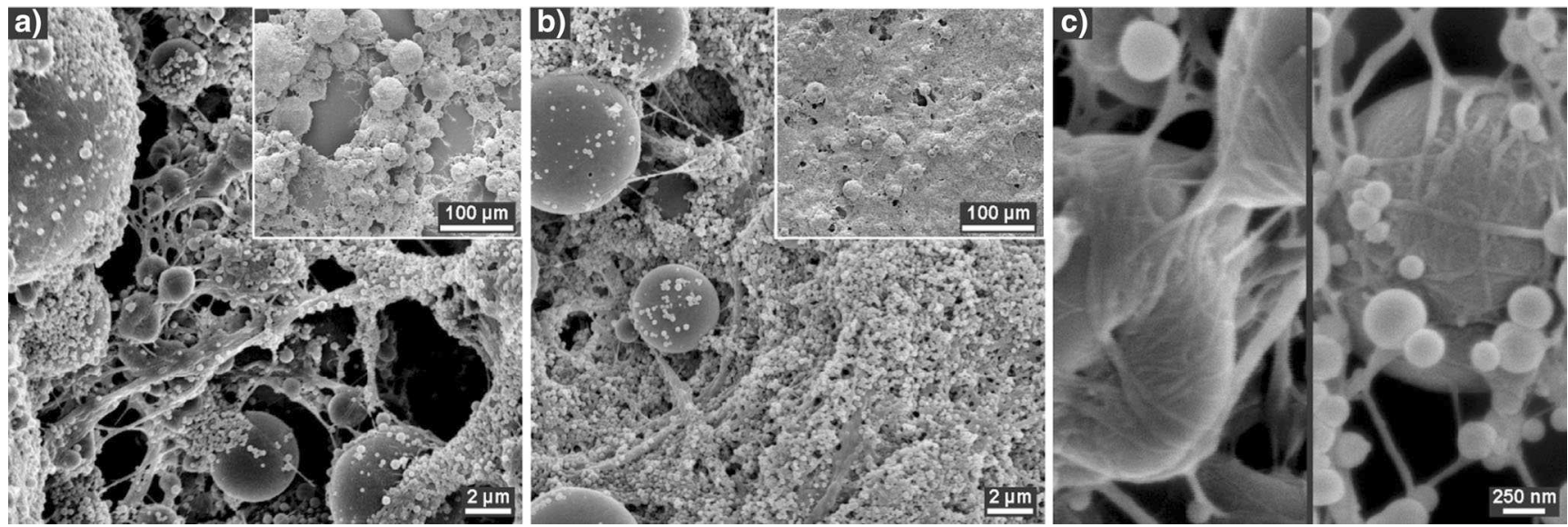

Fig. 7 SEM images of NFC/PS suspensions from control experiments (initiator: AIBN): polymerization of PS instead of PMMA in presence of $\mathrm{NFC}$ at NFC concentrations of 0.05 (a) and 0.5 (b) $\mathrm{wt} \%$ and magnification of large PS beads enwrapped and interconnected by NFC fibrils (c); images a, b can be compared with the SEM images in Fig. 2a, d 
contrast to the homogenously distributed PMMA spheres, which were polymerized in the presence of NFC (Fig. 2). It indicates that the presence of NFC during the heterophase polymerization of MMA is essential to form PMMA spheres with uniform diameters and regular distribution along the NFC network. A similar stabilizing effect was suggested for the heterophase polymerization of MMA in presence of silica particles under static conditions, where adsorption of oligomeric PMMA in the early stage of the polymerization onto the silica surfaces was assumed [6].

\section{Role of monomer}

The obtained NFC/PMMA structures shown in Fig. 2 were much different and unique compared to other reported products prepared by heterophase polymerization in presence of NFC or shorter nanocellulose crystals (NCC). There, mainly styrene was used in miniemulsion polymerization and polystyrene (PS) spheres in the size range of few micrometers were obtained, for instance, in [20-22]. In contrast, structures quite comparable to ours were obtained, when the water-soluble monomer pyrrole was polymerized to polypyrrole (PPy) in presence of bacterial cellulose $(\mathrm{BC})$ via oxidative polymerization in water [43, 44]. These results with PPy indicate that our NFC/PMMA structures were also arising due to the comparably high solubility of MMA in water (1.5 $\left.\mathrm{wt}^{\mathrm{O}} \%\right)$.

For this reason, a control experiment was conducted, which supports the crucial role of the MMA solubility in the NFC/PMMA heterophase polymerization reaction. Hence, MMA was replaced by styrene, which exhibits a marginal solubility of $0.024 \mathrm{wt} \%$ in water.

Predominantly large PS beads with diameters as large as $60 \mu \mathrm{m}$ were visible on SEM images, especially at a lower NFC concentration (Fig. a7). In line with our assumption, styrene is rather dispersed than dissolved in the aqueous phase, and thus, comparably big styrene droplets were then transformed to big PS beads due to monomer droplet initiation and polymerization. At increasing NFC concentration, styrene is better dispersed within the NFC network structures, which reduced the amount of large PS beads (Fig. 7b). Small PS spheres in nanometer range $(\approx 200 \mathrm{~nm})$ were found as well, which most probably emerged due to the very low, but still present water solubility of styrene. Interestingly, the large PS beads were partly enwrapped and interconnected by the NFC fibrils (Fig. 7c). Such structures were actually expected in the beginning of this study with PMMA, since similar assemblies were obtained as explained above, e.g., in [22], where styrene was used. They are explained with Pickering-like interactions between the NFC and the styrene or PS, respectively.

\section{Conclusions}

Nanoscale PMMA spheres homogeneously distributed within the NFC network were produced via heterophase polymerization of MMA in presence of NFC. The PMMA spheres adhered to the NFC fibrils and attractive interactions such as van der Waals interactions as well as hydrogen bonds between the NFC and the MMA (before polymerization) or PMMA (after polymerization), respectively, are reasonable. The appearance of the polymerized NFC/PMMA products was further strongly influenced by several parameters of the polymerization reaction conditions, which was supported by different control experiments:

- The static reaction condition was decisive for the formation of regularly distributed PMMA spheres, since the weak attractive forces between the NFC and MMA before polymerization were destroyed during stirring.

- The oil-soluble initiator AIBN favored the formation of PMMA spheres, which adhered to the NFC; no or only few non-bounded PMMA spheres were formed. In contrast, non-bounded PMMA spheres were obtained with the anionic initiator KPS, due to polymerization initiation in the aqueous phase. No efforts were done to investigate how strong the final PMMA spheres were bounded to the NFC.

- NFC served as stabilizer for the formation of PMMA spheres during the polymerization. Physical blending of pre-polymerized PMMA with NFC resulted in less homogeneous structures. The ability of NFC fibrils to interconnect with each other and, thus, the formation of flocks and high viscosity suspensions were important to inhibit phase separation of the monomer during the polymerization reaction and to promote a homogeneous distribution of PMMA spheres within the network.

- The slight solubility of the monomer MMA was crucial for the formation of the obtained nanoscale PMMA spheres rather than formation of initially expected large, NFC covered polymer particles.

Acknowledgments This work was financially supported by the Swiss National Science Foundation (SNF) within the National Research Programme 66 (NRP 66) "Resource Wood" Project-Nr 406640 136558). We kindly thank Beatrice Fischer for performing TGA measurements. We also thank Zellstoff Stendal for providing the cellulose raw material.

\section{Compliance with ethical standards}

Conflict of interest The authors declare that they have no conflict of interest. 


\section{References}

1. Chevalier Y, Bolzinger M-A (2013) Emulsions stabilized with solid nanoparticles: Pickering emulsions. Colloid Surf A 439:23-34. doi: 10.1016/j.colsurfa.2013.02.054

2. Ramsden W (1903) Separation of solids in the surface-layers of solutions and 'suspensions'. Proc R Soc Lond 72(477-486):156164. doi:10.1098/rspl.1903.0034

3. Pickering SU (1907) Emulsions. J Chem Soc Trans 91:2001-2021. doi:10.1039/CT9079102001

4. Binks BP (2002) Particles as surfactants - similarities and differences. Curr Opin Colloid Interface Sci 7(1-2):21-41. doi:10.1016/ S1359-0294(02)00008-0

5. Schrade A, Landfester K, Ziener U (2013) Pickering-type stabilized nanoparticles by heterophase polymerization. Chem Soc Rev 42(16):6823-6839. doi:10.1039/c3cs60100e

6. Kawano S, Sei A, Kunitake M (2010) Sparsely-distributed silica/ PMMA composite particles prepared by static polymerization in aqueous silica dispersion. J Colloid Interface Sci 352(2):348-353. doi:10.1016/j.jcis.2010.08.061

7. Chen JH, Cheng C-Y, Chiu W-Y, Lee C-F, Liang N-Y (2008) Synthesis of $\mathrm{ZnO} /$ polystyrene composites particles by Pickering emulsion polymerization. Eur Polym J 44(10):3271-3279. doi:10. 1016/j.eurpolymj.2008.07.023

8. García-Chávez KI, Hernández-Escobar CA, Flores-Gallardo SG, Soriano-Corral F, Saucedo-Salazar E, Zaragoza-Contreras EA (2013) Morphology and thermal properties of clay/PMMA nanocomposites obtained by miniemulsion polymerization. Micron 49: 21-27. doi:10.1016/j.micron.2013.02.007

9. Xu Z, Xia A, Wang C, Yang W, Fu S (2007) Synthesis of raspberrylike magnetic polystyrene microspheres. Mater Chem Phys 103(23):494 499. doi:10.1016/j.matchemphys.2007.02.074

10. Walther A, Hoffmann M, Müller AHE (2008) Emulsion polymerization using Janus particles as stabilizers. Angew Chem Int Ed 47(4):711-714. doi:10.1002/anie.200703224

11. Percy MJ, Barthet C, Lobb JC, Khan MA, Lascelles SF, Vamvakaki M, Armes SP (2000) Synthesis and characterization of vinyl polymer-silica colloidal nanocomposites. Langmuir 16(17):69136920. doi:10.1021/la0004294

12. Jeng J, Chen T-Y, Lee C-F, Liang N-Y, Chiu W-Y (2008) Growth mechanism and $\mathrm{pH}$-regulation characteristics of composite latex particles prepared from Pickering emulsion polymerization of aniline/ZnO using different hydrophilicities of oil phases. Polymer 49(15):3265-3271. doi:10.1016/j.polymer.2008.05.027

13. Salas C, Nypelö T, Rodriguez-Abreu C, Carrillo C, Rojas OJ (2014) Nanocellulose properties and applications in colloids and interfaces. Curr Opin Colloid Interface Sci 19(5):383-396. doi:10.1016/j. cocis.2014.10.003

14. Lam S, Velikov KP, Velev OD (2014) Pickering stabilization of foams and emulsions with particles of biological origin. Curr Opin Colloid Interface Sci 19(5):490-500. doi:10.1016/j.cocis. 2014.07.003

15. Lee K-Y, Bismarck A, Stoyanov SD, Paunov VN (2014) Colloidal and nanocellulose-stabilized emulsions. In: Handbook of green materials, pp. 185-196. doi:10.1142/9789814566469_0042

16. Kondo T (2007) Nematic ordered cellulose: its structure and properties. In: Brown Jr RM, Saxena I (eds) Cellulose: molecular and structural biology. Springer, Heidelberg, pp. 285-305. doi:10.1007/ 978-1-4020-5380-1 16

17. Ben Elmabrouk A, Thielemans W, Dufresne A, Boufi S (2009) Preparation of poly(styrene-co-hexylacrylate)/cellulose whiskers nanocomposites via miniemulsion polymerization. J Appl Polym Sci 114(5):2946-2955. doi:10.1002/app.30886
18. Ben Mabrouk A, Rei Vilar M, Magnin A, Belgacem MN, Boufi S (2011) Synthesis and characterization of cellulose whiskers/ polymer nanocomposite dispersion by mini-emulsion polymerization. J Colloid Interface Sci 363(1):129-136. doi:10.1016/j.jcis. 2011.07.050

19. Mabrouk AB, Salon MCB, Magnin A, Belgacem MN, Boufi S (2014) Cellulose-based nanocomposites prepared via miniemulsion polymerization: understanding the chemistry of the nanocellulose/matrix interface. Colloid Surf A 448:1-8. doi:10. 1016/j.colsurfa.2014.01.077

20. Kalashnikova I, Bizot H, Cathala B, Capron I (2011) New Pickering emulsions stabilized by bacterial cellulose nanocrystals. Langmuir 27(12):7471-7479. doi:10.1021/la200971f

21. Kalashnikova I, Bizot H, Cathala B, Capron I (2011) Modulation of cellulose nanocrystals amphiphilic properties to stabilize oil/water interface. Biomacromolecules 13(1):267-275. doi:10.1021/ bm201599j

22. Kalashnikova I, Bizot H, Bertoncini P, Cathala B, Capron I (2013) Cellulosic nanorods of various aspect ratios for oil in water Pickering emulsions. Soft Matter 9(3):952-959. doi:10.1039/ c2sm26472b

23. $\mathrm{Hu} \mathrm{Z}$, Patten T, Pelton R, Cranston ED (2015) Synergistic stabilization of emulsions and emulsion gels with water-soluble polymers and cellulose nanocrystals. ACS Sustain Chem Eng 3(5):10231031. doi:10.1021/acssuschemeng.5b00194

24. Nypelö T, Rodriguez-Abreu C, Kolen'ko YV, Rivas J, Rojas OJ (2014) Microbeads and hollow microcapsules obtained by selfassembly of Pickering magneto-responsive cellulose nanocrystals. ACS Appl Mater Interfaces 6(19):16851-16858. doi:10.1021/ am504260u

25. Zoppe JO, Venditti RA, Rojas OJ (2012) Pickering emulsions stabilized by cellulose nanocrystals grafted with thermo-responsive polymer brushes. J Colloid Interface Sci 369(1):202-209. doi:10. 1016/j.jcis.2011.12.011

26. Winuprasith T, Suphantharika M (2013) Microfibrillated cellulose from mangosteen (Garcinia mangostana L.) rind: preparation, characterization, and evaluation as an emulsion stabilizer. Food Hydrocoll 32(2):383-394. doi:10.1016/j.foodhyd.2013.01.023

27. Xhanari K, Syverud K, Stenius P (2011) Emulsions stabilized by microfibrillated cellulose: the effect of hydrophobization, concentration and O/W ratio. J Dispers Sci Technol 32(3):447-452. doi:10. 1080/01932691003658942

28. Littunen K, Hippi U, Johansson L-S, Österberg M, Tammelin T, Laine J, Seppälä J (2011) Free radical graft copolymerization of nanofibrillated cellulose with acrylic monomers. Carbohydr Polym 84(3):1039-1047. doi:10.1016/j.carbpol.2010.12.064

29. Nikfarjam N, Taheri Qazvini N, Deng Y (2015) Surfactant free Pickering emulsion polymerization of styrene in $\mathrm{w} / \mathrm{o} / \mathrm{w}$ system using cellulose nanofibrils. Eur Polym J 64:179-188. doi:10. 1016/j.eurpolymj.2015.01.007

30. Grüneberger F, Künniger T, Zimmermann T, Arnold M (2014) Rheology of nanofibrillated cellulose/acrylate systems for coating applications. Cellulose 21(3):1313-1326. doi:10.1007/s10570014-0248-9

31. Schramm LL (2006) 2 dispersion and dispersed species characterization. In: Emulsions, foams, and suspensions. Wiley-VCH Verlag GmbH \& Co. KGaA, Weinheim, pp. 13-51. doi:10.1002/ $3527606750 . c h 2$

32. Eyholzer C, Bordeanu N, Lopez-Suevos F, Rentsch D, Zimmermann T, Oksman K (2010) Preparation and characterization of waterredispersible nanofibrillated cellulose in powder form. Cellulose 17(1):19-30. doi:10.1007/s10570-009-9372-3

33. Karppinen A, Saarinen T, Salmela J, Laukkanen A, Nuopponen M, Seppälä J (2012) Flocculation of microfibrillated cellulose in shear flow. Cellulose 19(6):1807-1819. doi:10.1007/s10570-012-9766-5 
34. Tatsumi D, Ishioka S, Matsumoto T (2002) Effect of fiber concentration and axial ratio on the rheological properties of cellulose fiber suspensions. Nihon Reoroji Gakkaishi 30(1):27-32. doi:10.1678/ rheology. 30.27

35. Abend S, Bonnke N, Gutschner U, Lagaly G (1998) Stabilization of emulsions by heterocoagulation of clay minerals and layered double hydroxides. Colloid Polym Sci 276(8):730-737. doi:10.1007/ s003960050303

36. Liu H, Geng S, Hu P, Qin Q, Wei C, Lv J (2015) Study of Pickering emulsion stabilized by sulfonated cellulose nanowhiskers extracted from sisal fiber. Colloid Polym Sci 293(3):963-974. doi:10.1007/ s00396-014-3484-5

37. Sèbe G, Ham-Pichavant F, Pecastaings G (2013) Dispersibility and emulsion-stabilizing effect of cellulose nanowhiskers esterified by vinyl acetate and vinyl cinnamate. Biomacromolecules 14(8):29372944. doi:10.1021/bm400854n

38. Hu Z, Ballinger S, Pelton R, Cranston ED (2015) Surfactantenhanced cellulose nanocrystal Pickering emulsions. J Colloid Interface Sci 439:139-148

39. Landfester K (2001) Polyreactions in miniemulsion. Macromol Rapid Commun 22(12):896-936. doi:10.1002/1521-3927(20010801) 22:12<896::AID-MARC896>3.0.CO;2-R
40. Van Herk AM (2013) Chemistry and technology of emulsion polymerisation, 2 edn. Wiley-VCH Verlag GmbH \& Co. KGaA, Weinheim

41. Egen M, Zentel R (2004) Surfactant-free emulsion polymerization of various methacrylates: towards monodisperse colloids for polymer opals. Macromol Chem Phys 205(11):1479-1488. doi:10. 1002/macp.200400087

42. Luna-Xavier JL, Bourgeat-Lami E, Guyot A (2001) The role of initiation in the synthesis of silica/poly(methyl methacrylate) nanocomposite latex particles through emulsion polymerization. Colloid Polym Sci 279(10):947-958. doi:10.1007/s003960100520

43. Muller D, Rambo CR, Recouvreux DOS, Porto LM, Barra GMO (2011) Chemical in situ polymerization of polypyrrole on bacterial cellulose nanofibers. Synth Met 161(1-2):106-111. doi:10.1016/j. synthmet.2010.11.005

44. Muller D, Rambo CR, Porto LM, Schreiner WH, Barra GMO (2013) Structure and properties of polypyrrole/bacterial cellulose nanocomposites. Carbohydr Polym 94(1):655-662. doi:10.1016/j.carbpol.2013.01. 041 